\title{
Mensaje del Presidente de la Sociedad Mexicana de Neumología y Cirugía de Tórax 2019-2021
}

\author{
Message from the President of the Mexican Society \\ of Pneumology and Thorax 2019-2021
}

\author{
Luis Adrián Rendón Pérez*
}

*Presidente de la Sociedad Mexicana de Neumología y Cirugía de Tórax.

Estimados socios y amigos de la Sociedad Mexicana de Neumología y Cirugía de Tórax:

Aprovecho este espacio en nuestra revista Neumología y Cirugía de Tórax (NCT), porque quiero que mis primeras palabras hacia ustedes sean de agradecimiento por el gran honor que me concedieron al permitirme trabajar desde la Presidencia por el engrandecimiento de nuestra querida Sociedad Mexicana de Neumología y Cirugía de Tórax (SMNyCT) durante el período 2019-2021. Les reitero mi compromiso de poner mis habilidades y mi máximo esfuerzo a su servicio, para que juntos hagamos de nuestra especialidad, una especialidad que sea reconocida como lo merece y que, en consecuencia, sea más influyente en la mejora de la atención de los pacientes con padecimientos respiratorios de México. La SMNyCT está cumpliendo 80 años de estar promoviendo la salud respiratoria y es mi intención que potenciemos nuestra influencia en la comunidad médica y la sociedad en general.

No quiero dejar pasar la oportunidad de felicitar a mis dos predecesores en la presidencia, al Dr. Juan Carlos Vázquez García y a la Dra. Mayra Edith Mejía Ávila quienes con inteligencia trabajaron conjuntamente durante sus administraciones creando proyectos a largo plazo; algunos de éstos ya consolidados y otros, en desarrollo, pero todos exitosos. El equipo de apoyo administrativo es inmejorable. Tengo la firme convicción de respetar lo que ha funcionado y de mejorarlo, si es posible. No podemos estar empezando de cero cada vez que ocurra un cambio de administración.

Correspondencia:

Dr. Luis Adrián Rendón Pérez

Presidente de la SMNYCT.

Correo electrónico: adrianrendon@hotmail.com

Trabajo recibido: 17-VII-2019; aceptado: 17-VII-2019.
Históricamente las enfermedades respiratorias han sido una amenaza permanente para la humanidad causando un gran número de muertes. A pesar de eso, siguen siendo menospreciadas como un problema de salud pública. En la actualidad, de las 10 principales causas de muerte identificadas por la Organización Mundial de la Salud, cuatro corresponden a enfermedades respiratorias: enfermedad pulmonar obstructiva crónica, cáncer de pulmón, infecciones respiratorias y tuberculosis. Si las consideramos juntas como «Enfermedades respiratorias», ocuparían el primer lugar. Además de su letalidad, las enfermedades respiratorias son muy prevalentes y los sistemas de salud no están preparados, ni para diagnosticarlas oportunamente, ni para manejarlas en el nivel primario de atención, que es donde están la mayoría de los enfermos. El escenario se hace más sombrío con los problemas crecientes de contaminación, cambio climático, migración y pobreza, entre otros, situaciones todas que complican el control a gran escala de dichos padecimientos.

Esta problemática nos abre la oportunidad de que siendo la Sociedad que agrupa a los mejores especialistas en salud respiratoria de México, asumamos la responsabilidad y tomemos la iniciativa de cooperar en todos los frentes en la lucha contra las enfermedades respiratorias.

Son varias las iniciativas que quiero compartir con ustedes. Bajo el lema de «Neumología para todos» y con una planeación adecuada, hay que lograr la proyección de nuestra Sociedad hacia la medicina general y a otras especialidades, hacia las autoridades sanitarias y también a la comunidad. Debemos hacer difusión de que somos los expertos en el manejo de enfermedades tan comunes como el asma y las infecciones respiratorias, entre otras. Busquemos una transformación interna en la que respetemos la tradición. La SMNyCT alberga médicos ilustres que con sus iniciativas han cimentado nuestra Sociedad, permitiendo 
que se convirtiera en lo que hoy es. El reto actual es ser una Sociedad capaz de influir en el ámbito de la salud pública. Anticipo que la tarea no será nada fácil y que llevará años por lo que es imperativo empezar a la brevedad.

A la Mesa Directiva de la SMNyCT le queda claro que es su responsabilidad seguir facilitando la educación médica continua de sus socios pero ahora, además, deberá buscar los caminos para que los conocimientos de nuestros socios puedan ser aprovechados por otros médicos y por los pacientes que así lo requieran. La Neumología debe estar dispersa por todo el país y no concentrarse sólo en las grandes ciudades. En cada estado, en cada ciudad, en cada lugar donde haya enfermos respiratorios, debe haber médicos que tengan los conocimientos elementales de los principales padecimientos pulmonares.

Por lo anterior, hemos planeado actividades en los estados del país. Estarán dirigidas a médicos de primer contacto y serán realizadas en conjunto con las autoridades sanitarias locales. Los miembros de los Capítulos de la SMNyCT, tendrán una participación activa y preponderante. También queremos llevar a cabo brigadas médicas neumológicas en comunidades rurales, en las que se han identificado grupos vulnerables afectados por padecimientos respiratorios. Todos ustedes estarán cordialmente invitados a ser protagonistas. Es esperado que la repercusión que causarán estas actividades le dará visibilidad a nuestra Sociedad, eso generará proyección y de la mano vendrá la facultad de hacer abogacía por la salud respiratoria.

Para lo anterior, es indispensable tener credibilidad y eso sólo se logra manteniendo una preparación científica de alto nivel que raye en la excelencia. Eventos académicos de temas específicos con expertos mundiales, en conjunto con nuestras sociedades hermanas internacionales, serán una herramienta con ese fin. Y por supuesto, nuestro próximo congreso anual en la ciudad de Monterrey del 13 al 17 de abril de 2020 (www.congresoneumologia2020.mx) que debe no sólo mantener su categoría de ser uno de los mejores de América sino, además, buscar pasar del plano internacional al mundial. Estaremos trabajando sin cansancio y con gran ilusión para cumplir con esta expectativa y hacer del congreso un evento memorable. Los Jefes de Sección que participarán en esta tarea están distribuidos por todo el país. Son un conjunto de gente experimentada trabajando con gente joven, algunos de ellos viviendo su primera experiencia en esta actividad. Todos ellos tienen en común ser líderes de opinión y exitosos en su profesión. Soy un convencido de que los jóvenes deben tomar retos y quienes ya nos enfrentamos antes a estos desafíos, les debemos dar oportunidades de crecer. Recordemos que la Neumología y nuestra Sociedad en poco tiempo quedarán en sus manos.

Nuestra revista que también cumple 80 años, seguirá teniendo un cuidado especial y buscaremos su modernización y su inscripción en los índices más reconocidos.

Queridos amigos, los invito a ser parte de nuestros proyectos. Recordemos que la SMNyCT no es la Mesa Directiva, la Sociedad somos todos sus miembros. Su cooperación y su retroalimentación son invaluables y necesarios para poder trascender.

Les envío un cordial abrazo y espero verlos pronto en alguno de nuestros próximos eventos.

Su amigo, Adrián 